\title{
Relaxation of the MCL after an Open-Wedge High Tibial Osteotomy results in decreasing contact pressures of the knee over time
}

\author{
N. van Egmond ${ }^{1}$ G. Hannink ${ }^{2}$ - D. Janssen ${ }^{2}$ - A. C. Vrancken ${ }^{2}$ N. Verdonschot ${ }^{2,3}$. \\ A. van Kampen ${ }^{1}$
}

Received: 17 August 2016 / Accepted: 16 January 2017 / Published online: 15 February 2017

(C) The Author(s) 2017. This article is published with open access at Springerlink.com

\begin{abstract}
Purpose The objective of this study was to investigate the effect of a medial open-wedge osteotomy (OWO) and the release of the superficial medial collateral ligament (MCL) on the tibiofemoral cartilage pressure, the MCL tension and the valgus laxity of the knee.

Methods Seven fresh-frozen, human cadaveric knees were used. Medial and lateral mean contact pressure (CP), peak contact pressure (peakCP), and contact area (CA) were measured using a pressure-sensitive film (I-Scan; Tekscan, Boston, MA). The MCL tension was measured using a custom-made device. These measurements were continuously recorded for $5 \mathrm{~min}$ after an OWO of $10^{\circ}$. After the osteotomy, the valgus laxity was measured with
\end{abstract}

N. van Egmond

Nienkevanegmond@hotmail.com

G. Hannink

Gerjon.hannink@radboudumc.nl

D. Janssen

Dennis.janssen@radboudumc.nl

A. C. Vrancken

Actvrancken@gmail.com

N. Verdonschot

Nico.verdonschot@ radboudumc.nl

A. van Kampen

Albertvankampen@gmail.com

1 Department of Orthopaedics, Radboud University Medical Centre, P.O. box 9101, 6500 HB Nijmegen, The Netherlands

2 Orthopaedic Research Laboratory, Radboud University Medical Center, P.O. box 9101, 6500 HB Nijmegen, The Netherlands

3 Laboratory of Biomechanical Engineering, University of Twente, P.O. box 217, 7500 AE Enschede, The Netherlands a handheld Newtonmeter. For one knee, the measurements were continued for $24 \mathrm{~h}$. At the end, a complete release of the superficial MCL was performed and the measurements were repeated at $10^{\circ}$.

Results There was relaxation of the MCL after the osteotomy; the tension dropped in 5 min with $10.7 \%$ (mean difference $20.5 \mathrm{~N}$ (95\% CI 16.1-24.9)), and in $24 \mathrm{~h}$, the tension decreased by $24.2 \%$ (absolute difference $38.8 \mathrm{~N}$ ) (one knee). After the osteotomy, the mean $\mathrm{CP}$, peakCP and $\mathrm{CA}$ increased in the medial compartment (absolute difference $0.17 \mathrm{MPa}$ (95\% CI $0.14-0.20), 0.27 \mathrm{MPa}(95 \% \mathrm{CI}$ 0.24-0.30), 132.9 $\mathrm{mm}^{2}$ (95\% CI 67.7-198.2), respectively), and decreased in the lateral compartment (absolute difference $0.02 \mathrm{MPa}(95 \% \mathrm{CI} 0.03-0.01), 0.08 \mathrm{MPa}(95 \% \mathrm{CI}$ $0.11-0.04), 47.0 \mathrm{~mm}^{2}$ (95\% CI -105.8 to 11.8$)$, respectively). Only after a release of the superficial MCL, the mean $\mathrm{CP}$, peak $\mathrm{CP}$ and $\mathrm{CA}$ significantly decreased in the medial compartment (absolute difference 0.17, 0.27 MPa, $119.8 \mathrm{~mm}^{2}$, respectively), and increased in the lateral compartment (absolute difference 0.02, $0.11 \mathrm{MPa}, 52.4 \mathrm{~mm}^{2}$, respectively). After the release of the superficial MCL, a mean increase of $7.9^{\circ}$ [mean difference $-0.1^{\circ}$ (95\% CI -1.9 to 1.6)] of the valgus laxity was found.

Conclusions A release of the superficial MCL helps achieve the goal of reducing medial cartilage pressure in an OWO. There was considerable relaxation of the MCL after an OWO that resulted in a decrease of the mean CP in the medial and lateral compartments of the knee over time. However, cartilage pressure shifted from the medial to the lateral compartment only after release of the superficial MCL. The release of the superficial MCL caused a significant increase in the valgus laxity, which could influence stability after an OWO.

Level of evidence $\mathrm{I}$. 
Keywords Open-wedge high tibial osteotomy .

Tibiofemoral cartilage pressure $\cdot$ Release medial collateral ligament $\cdot$ Valgus laxity $\cdot$ Biomechanical study

\section{Introduction}

An open-wedge osteotomy (OWO) is a successful treatment in patients with medial knee osteoarthritis (OA) and a varus leg alignment $[4,18]$. An OWO unloads the medial compartment and shifts loading of the knee to the lateral compartment $[1,9,17]$.

In an OWO, the medial proximal tibia must be exposed; however, the superficial medial collateral ligament (MCL) overlies this area. The superficial MCL can be left intact by elevating it sub-periostally, or it can be partially or completely released from its distal insertion [8, 11]. In literature, there is a debate as to whether or not to release the MCL when performing an OWO. Agneskircher et al. [1] concluded in their biomechanical study that if the MCL is not released after an OWO, the contact pressure in the medial compartment is even higher than in the lateral compartment. On the other hand, a release of the MCL has been shown to create a significant valgus instability [11].

It is known that ligaments show relaxation over time [5, 16], i.e. the tension in a ligament decreases over time with a constant strain. This is primarily due to maintenance of the structure in a strained condition for some finite interval of time, hence, causing some amount of plastic strain. This should not be confused with creep, which is a constant state of stress with an increasing amount of strain. The largest relaxation occurs within the first six to eight hours. After this, the effect is much smaller [5]. Theoretically, relaxation of the MCL after an OWO would result in a decrease of the cartilage pressure in the medial compartment, and the release of the superficial MCL, on that account, may not be necessary. The relaxation of the MCL and its influence on cartilage pressure has not yet been investigated.

The purpose of the present study was to investigate (1) the effect of MCL relaxation after an OWO on the contact pressure (CP), peak contact pressure (peakCP) and contact area (CA), in the medial and lateral compartments, (2) the effect of a complete release of the superficial MCL after an OWO on the $\mathrm{CP}$, peakCP and CA in the medial and lateral compartments, and (3) the effect of a complete release of the superficial MCL after an OWO on the valgus laxity of the knee. It was hypothesised that (1) tension over the MCL gradually decreases, and correspondingly, the cartilage pressure in the medial compartment also decreases over time; (2) after a release of the superficial MCL, the $\mathrm{CP}$, peakCP and $\mathrm{CA}$ in the medial compartment decreases; (3) after a release of the superficial MCL, the valgus laxity of the knee increases.

\section{Materials and methods}

Seven fresh-frozen, human cadaveric left legs were used in this study (mean age 78.9-year old (range 64-90), four men). The tibia and fibula were left as long as possible, leaving enough space for the custom-made device. The femur was cut mid-way. The cadavers were thawed over $24 \mathrm{~h}$ and dissected with the removal of the skin and all subcutaneous tissue. The joints were opened through a medial parapatellar approach and the quadriceps, patella, patellar tendon and anterior capsule were removed. The medial and lateral collateral ligaments (MCL and LCL), the anterior and posterior cruciate ligaments (ACL and PCL) were left intact, as was the posterior joint capsule and the popliteus tendon. The menisci were resected. The joints were visually inspected for signs of previous operations, injuries and signs of osteoarthritis. There were no signs of previous operations and injuries. Four knees had no signs of OA, one knee had mild signs of OA, and two knees had severe signs of OA, without severe osteophyte formation. None of the knees had deformities. The tibia stump was embedded in cement in a custom-made device. The femur was embedded in cement in extension, and was kept in place during cementation using a Kirschner wire (Fig. 1).

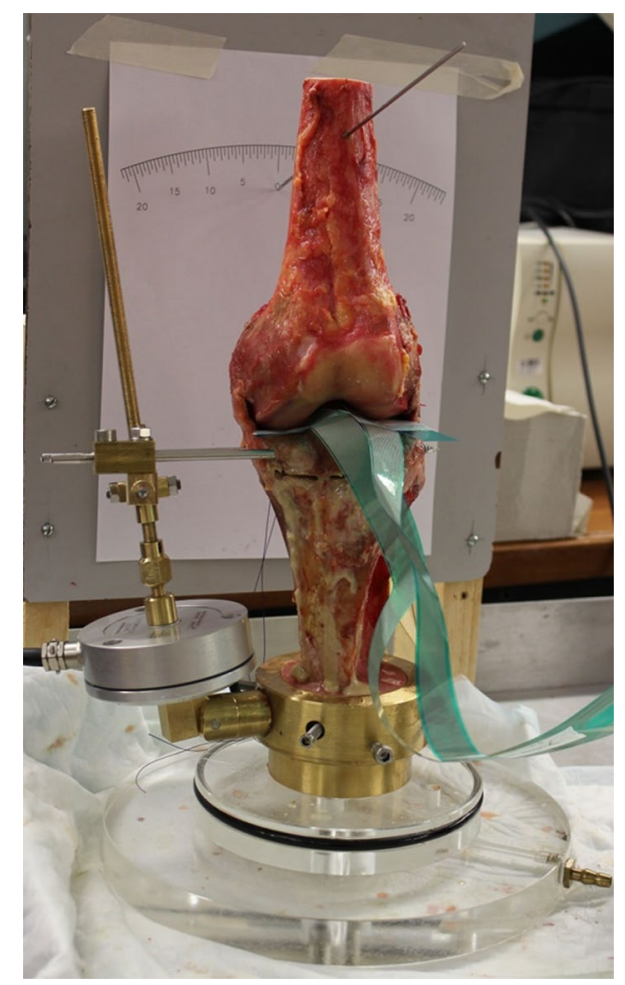

Fig. 1 Experimental set-up with the custom-made device 


\section{Osteotomy}

A monoplanar medial open-wedge high tibial osteotomy was carried out, without a release of the MCL. A Kirschner wire was inserted parallel to the joint line, just proximal to the tuberosity, directed to the fibular head. Along this wire the osteotomy was performed, leaving 10 millimetres of the lateral cortex intact. The osteotomy was created using a custom-made device, which was fixed to the proximal part of the osteotomy by placing screws anterior and posterior to the MCL, parallel to the osteotomy gap (Fig. 1). The gap was opened gradually until the desired osteotomy angle of $10^{\circ}$ was reached, with one winding being equal to one millimetre. The number of millimetres c.q. windings was calculated by measuring the distance of the screw and the known desired angle $\left(10^{\circ}\right)$.

\section{MCL tension}

The custom-made device included a tensiometer incorporated within a $5 \mathrm{kN}$ force transducer (Burster 8531-5000, Burster sensors and precision measurement, Gernsbach, Germany) that was used to measure the force that was produced by the MCL (MCL tension) during the opening of the osteotomy gap. The force transducer has an accuracy of $\leq \pm 0.15 \%$ (or $\leq \pm 7.5 \mathrm{~N}$ ). In Fig. 1, the experimental set-up is shown, with the custom-made device including the tensiometer on the left side of the cadaver. The MCL tension was measured continuously for $5 \mathrm{~min}$ after an osteotomy of $10^{\circ}$ in full extension. For one knee, the measurements were continued for $24 \mathrm{~h}$.

\section{Tibiofemoral cartilage pressure}

A pressure-sensitive film [I-Scan Pressure Mapping Sensor 4000 (Tekscan, Boston, MA)] was inserted into the medial and lateral tibiofemoral joint and fixated to the posterior capsule using sutures. For protection of the sensor films, a thin piece of foil was placed over both sides of the sensor [20]. Before insertion into the joints, the sensors were preconditioned and calibrated using custom-made loading blocks in a mechanical testing system, as described in detail in other studies [19]. Care was taken to ensure that the sensors were seated on the cartilage without wrinkles. To ensure fixation, two sutures were placed through each sensor and the surrounding soft-tissue.

\section{Valgus laxity}

The valgus laxity was measured by applying a valgus moment of $2 \mathrm{~N} \mathrm{~m}$ to the proximal femur, using a handheld force gauge. The angular change, [measured on a protractor placed behind the femur (Fig. 1)] caused by the valgus moment was taken as a measure for the laxity.

\section{Measurements}

Baseline measurements of contact pressure (CP; in $\mathrm{MPa}$ ), peak contact pressure (peakCP; in $\mathrm{MPa}$ ) and contact area $\left(\mathrm{CA}\right.$; in $\mathrm{mm}^{2}$ ) of the medial and lateral compartments were continuously performed at $0^{\circ}$ gap opening, with the knee in full extension for a period of $5 \mathrm{~min}$. The MCL tension and the valgus laxity were also measured at baseline. Next, the osteotomy gap was opened to $10^{\circ}$, and $\mathrm{CP}$, peakCP and CA were measured again, as well as the MCL tension and the valgus laxity. The measurements (MCL tension, $\mathrm{CP}$, peakCP and CA) were performed continuously for $5 \mathrm{~min}$. For one knee, the measurements were continued for $24 \mathrm{~h}$ to assess the viscoelastic effects in the longer term.

After these measurements, a complete release of the superficial MCL was performed at the level of the osteotomy. All the measurements $(\mathrm{CP}$, peakCP and $\mathrm{CA}$, the MCL tension and valgus laxity) were repeated, at $0^{\circ}$ and $10^{\circ}$.

\section{Statistical analysis}

Descriptive statistics were used to summarise the data. Data were given as mean and standard deviation (SD) and differences were given as mean with $95 \%$ confidence intervals (CI). Linear mixed models were used to study the effect of condition (i.e. $0^{\circ}$ and $10^{\circ}$ valgus opening with and without MCL release) on CP, peakCP, CA, MCL tension and valgus laxity measurements. Patient/knee was treated as random factor. Regression parameter estimates were presented with their $95 \%$ CI. $p$ values $<0.05$ were considered statistically significant. The statistical analyses were performed using R version 3.2.2 (R Foundation for Statistical Computing, Vienna, Austria).

\section{Results}

\section{Tension produced by the MCL (MCL tension)}

At baseline, the MCL tension was $1.2 \mathrm{~N}$ (SD 3.8). Opening the osteotomy gap to $10^{\circ}$ caused an average increase in the MCL tension of 203 N (95\% CI 16.1-24.9) (Fig. 2). Monitoring the MCL tension after the osteotomy revealed relaxation of the MCL. In $5 \mathrm{~min}$, the tension dropped with $10.7 \%$ (mean difference $20.5 \mathrm{~N}$ (95\% CI 16.1-24.9)). In the knee that was continuously monitored for $24 \mathrm{~h}$, the MCL tension decreased with $24.2 \%$ (absolute difference $38.8 \mathrm{~N}$ ) within $24 \mathrm{~h}$. In that particular knee, within the first $5 \mathrm{~min}$, 


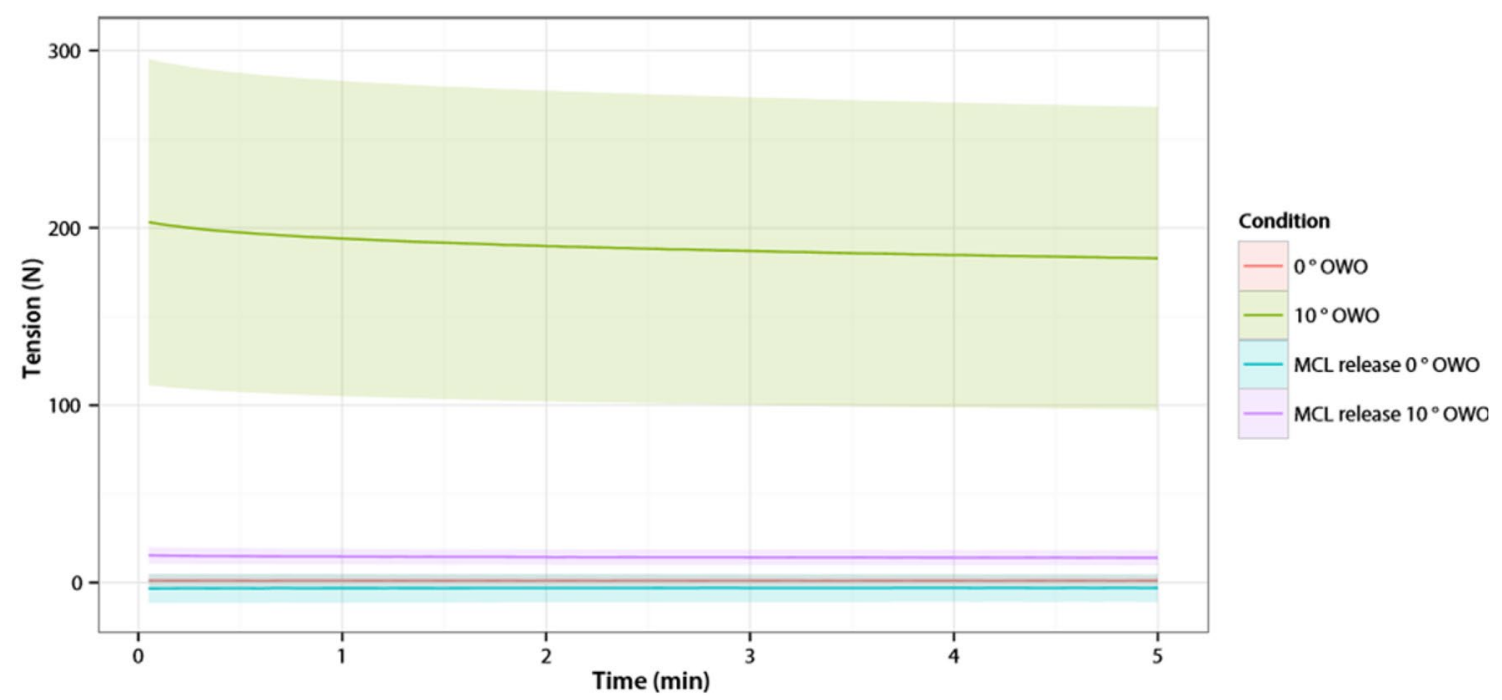

Fig. 2 Medial Collateral Ligament tension of all knees. On the $X$-axis is the time in minutes. On the $Y$-axis is the MCL tension in Newton (mean with $95 \%$ confidence interval)

Fig. 3 Medial Collateral Ligament tension in $24 \mathrm{~h}$. On the $X$-axis is the time in hours. On the $Y$-axis is the MCL tension in Newton

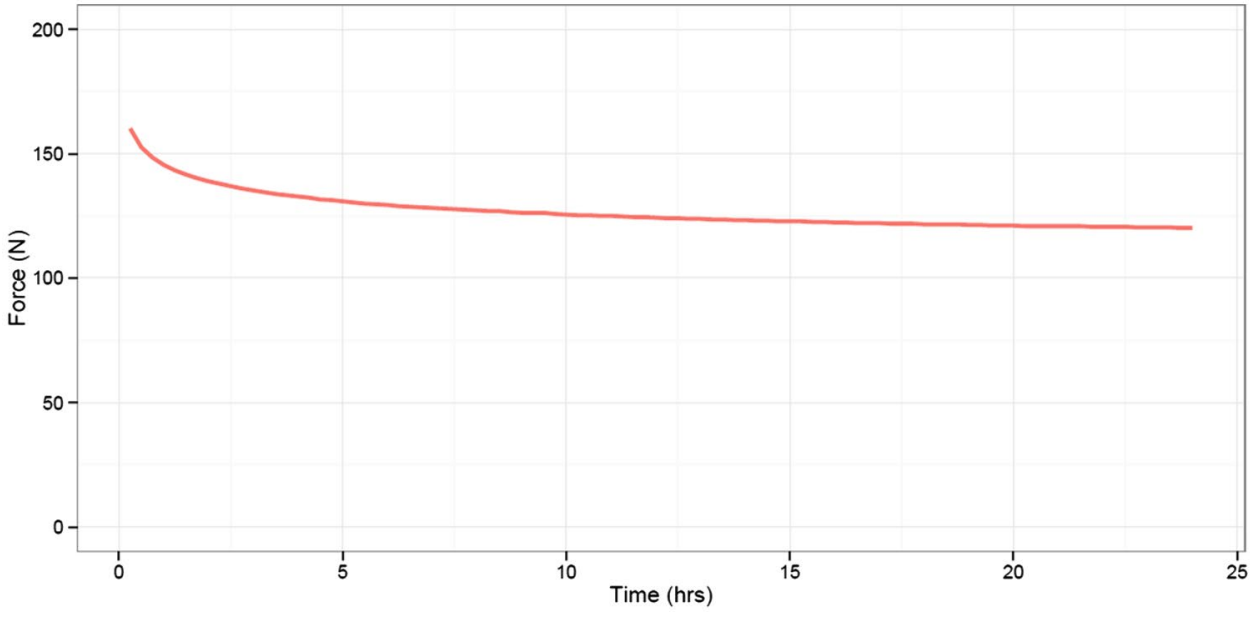

the tension decreased $2.1 \%$, and between $5 \mathrm{~min}$ and $24 \mathrm{~h}$ the tension decreased an additional 22.6\% (Fig. 3).

\section{Tibiofemoral cartilage pressures in the medial and lateral compartments}

After the osteotomy, the mean $\mathrm{CP}$, peakCP and $\mathrm{CA}$ in the medial compartment increased and in the lateral compartment decreased, compared to the situation without osteotomy (Table 1).

After the release of the superficial MCL, the mean CP, peakCP and CA significantly decreased in the medial compartment and significantly increased in the lateral compartment compared to the osteotomy situation. In this situation, the mean CP and peak CP in the lateral compartment increased relative to the medial compartment (Table 1).
Within the first 5 min after opening the osteotomy gap to $10^{\circ}$, the mean $\mathrm{CP}$ in the medial and lateral compartments slightly decreased (1.7\% (SD1.7) and 1.6\% (SD3.3), respectively) (Fig. 4).

In the knee that was continuously monitored for $24 \mathrm{~h}$, the CP decreased within $24 \mathrm{~h}$ with 11.3 and $10.5 \%$ in the medial and lateral compartments, respectively (Fig. 5).

\section{Valgus laxity}

Valgus laxity was unaffected by the osteotomy (mean difference $-0.1^{\circ}$ (95\% CI -1.9 to $1.6 ; p=$ n.s.)) alone. However, after the release of the superficial MCL, the laxity was significantly increased (mean difference $7.9^{\circ}$ (95\% CI $6.1-9.6 ; p<0.001)$ ) compared to the situation without OWO (Fig. 6). 
Table 1 Mean CP, peak CP, CA in the medial and lateral compartments-after 5 min compared to baseline and after MCL release

\begin{tabular}{|c|c|c|c|c|c|c|c|}
\hline Measurements & Baseline $^{\mathrm{a}}$ & Osteotomy $10^{\circ a}$ & MCL release $10^{\circ a}$ & $\begin{array}{l}\text { Absolute difference } \\
\text { osteotomy-baseline } \\
(95 \% \mathrm{CI})\end{array}$ & $P$ value & $\begin{array}{l}\text { Absolute difference } \\
\text { MCL release-osteot- } \\
\text { omy }(95 \% \text { CI) }\end{array}$ & $P$ value \\
\hline $\mathrm{CP}$ medial $(\mathrm{MPa})$ & $0.03(0.01)$ & $0.20(0.07)$ & $0.03(0.005)$ & $0.17(0.14-0.20)$ & $<0.001$ & $-0.17(-0.20$ to -0.13$)$ & $<0.001$ \\
\hline CP lateral (MPa) & $0.03(0.01)$ & $0.01(0.00)$ & $0.04(0.02)$ & $-0.02(-0.03$ to -0.01$)$ & 0.001 & $0.02(0.01-0.03)$ & $<0.001$ \\
\hline Peak CP medial $(\mathrm{MPa})$ & $0.10(0.04)$ & $0.38(0.05)$ & $0.11(0.05)$ & $0.27(0.24-0.30)$ & $<0.001$ & $-0.27(-0.30$ to -0.24$)$ & $<0.001$ \\
\hline Peak CP lateral (MPa) & $0.11(0.05)$ & $0.03(0.02)$ & $0.13(0.07)$ & $-0.08(-0.11$ to -0.04$)$ & $<0.001$ & $0.11(0.07-0.14)$ & $<0.001$ \\
\hline CA medial $\left(\mathrm{mm}^{2}\right)$ & $193.6(48.8)$ & $326.5(71.0)$ & $203.2(59.7)$ & $132.9(67.7-198.2)$ & $<0.001$ & $\begin{array}{l}-119.8(-185.3 \text { to } \\
-80.6)\end{array}$ & $<0.001$ \\
\hline CA lateral $\left(\mathrm{mm}^{2}\right)$ & $112.7(67.1)$ & $65.7(100.5)$ & $120.4(69.6)$ & $-47.0(-105.8$ to 11.8$)$ & N.S & $52.4(-9.2$ to 114.3$)$ & N.S \\
\hline
\end{tabular}

$C I$ confidence interval, $C P$ contact pressure, $C A$ contact area, $M C L$ medial collateral ligament, N.S. non-significant

${ }^{\mathrm{a}}$ Values given as mean (standard deviation)

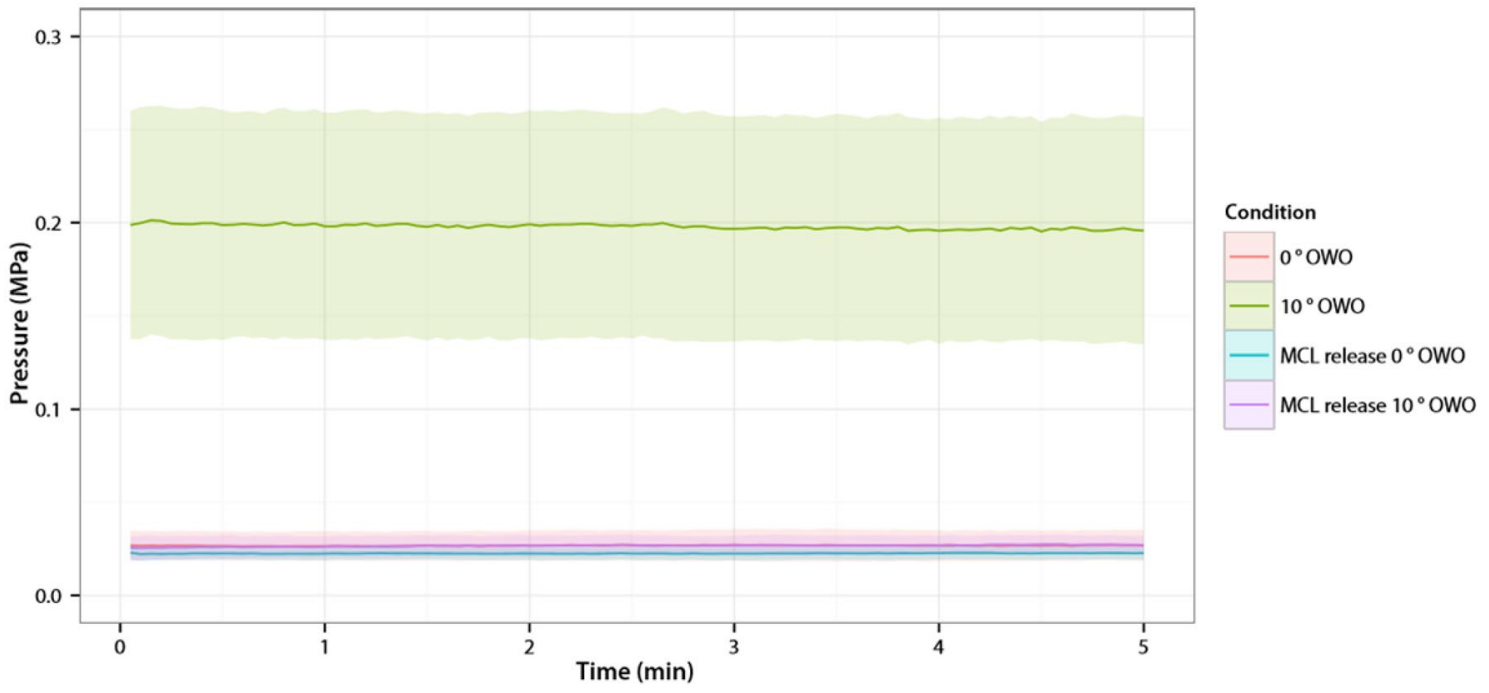

Fig. 4 Contact Pressure medial compartment of all knees. On the $X$-axis is the time in minutes. On the $Y$-axis is the Contact Pressure in the medial compartment in $\mathrm{MPa}$ (mean with $95 \%$ confidence interval)

\section{Discussion}

The most important findings of this study were as follows: Firstly, there was relaxation of the MCL after an OWO resulting in a decrease of the mean $\mathrm{CP}$ in the medial and lateral compartments of the knee over time. Secondly, after a complete release of the superficial MCL, there was a significant decrease of the mean $\mathrm{CP}$, peakCP and $\mathrm{CA}$ in the medial compartment and a significant increase of the mean $\mathrm{CP}$, peakCP and a non-significant decrease of the $\mathrm{CA}$ in the lateral compartment. Thirdly, a complete release of the superficial MCL gave a significant increase in valgus laxity.

After a release of the superficial MCL, a significant decrease of the mean $\mathrm{CP}$, peak $\mathrm{CP}$ and the $\mathrm{CA}$ in the medial compartment and a significant increase of the mean $\mathrm{CP}$, peakCP and non-significant increase of the $\mathrm{CA}$ in the lateral compartment were found. These results correspond to the study of Agneskircher et al. [1]. They investigated the $\mathrm{CP}$, peakCP and $\mathrm{CA}$ in the medial and lateral compartments with no release, a partial release and a complete release of the superficial MCL. They concluded that after a medial OWO, a complete release of the superficial MCL is required, as a shift of the cartilage pressure to the lateral compartment only occurs after this complete release. No other studies investigated the effect of a release of the superficial MCL after an OWO on the cartilage pressure. Our results confirmed the conclusions of Agneskircher et al. [1] that there is an upward mechanical lift of the medial part of the tibia plateau after an OWO without a release of the MCL, pressuring against the medial femoral condyle. They hypothesised that this leads to increased MCL tension and an associated increase of the cartilage pressure. Our results confirmed this hypothesis. We found that after an OWO of $10^{\circ}$ there was an increase in the 


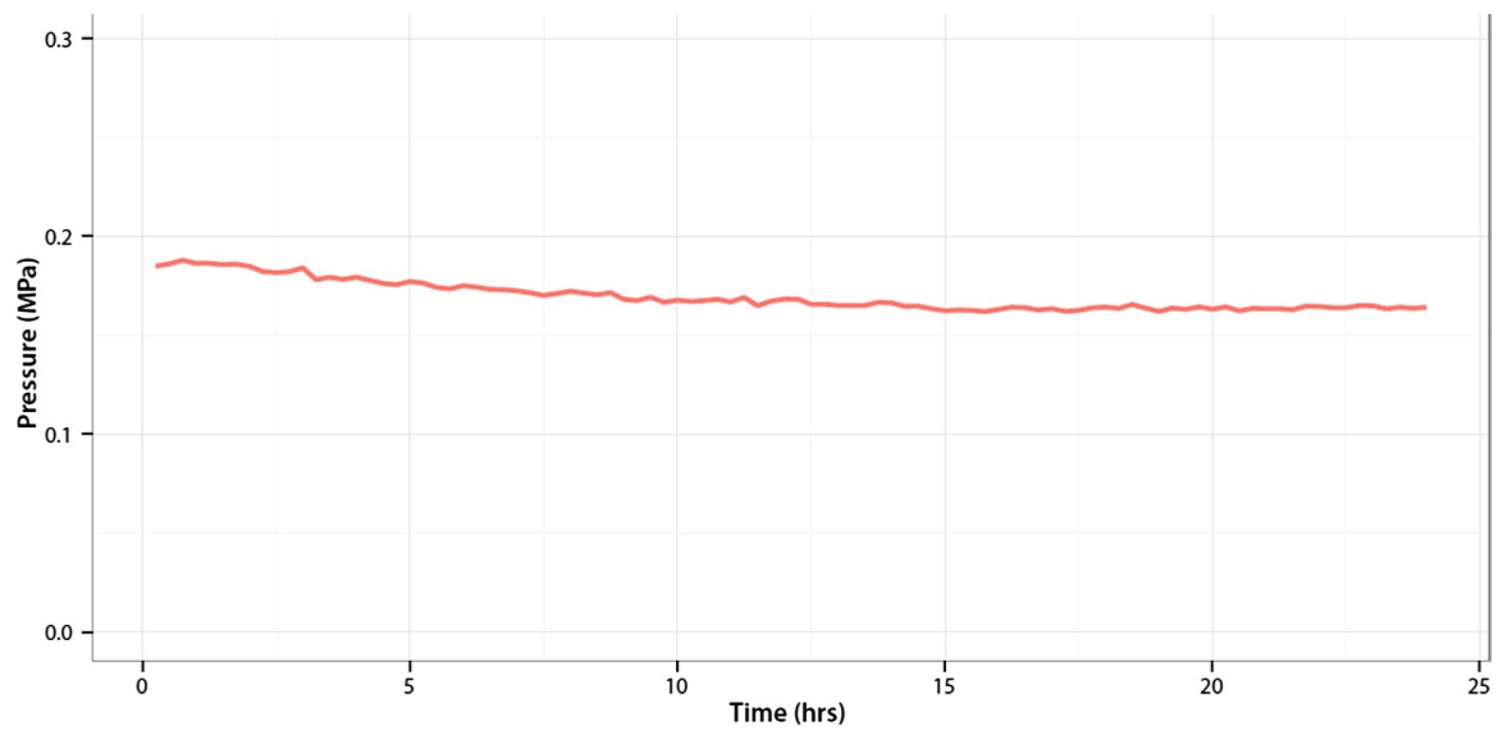

Fig. 5 Contact Pressure medial compartment in $24 \mathrm{~h}$. On the $X$-axis is the time in hours. On the $Y$-axis is the Contact Pressure in the medial compartment in $\mathrm{MPa}$

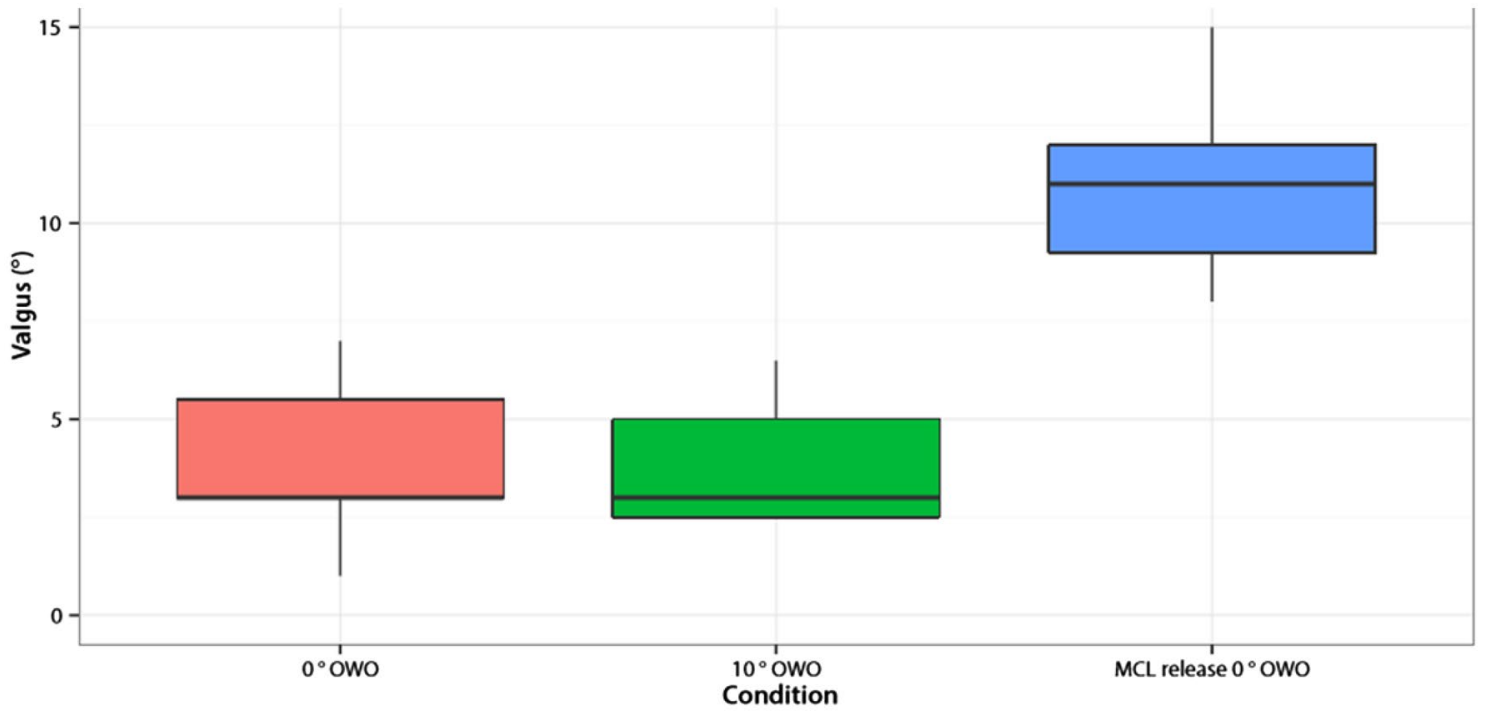

Fig. 6 Valgus stability of all knees. On the $X$-axis are the different conditions in which the valgus stability was measured. On the $Y$-axis is the amount of valgus in degrees

MCL tension of more than $200 \mathrm{~N}$. The MCL consists of two components: the superficial MCL and deep MCL [12]. The tensile strength of the superficial MCL and deep MCL has been reported to be approximately $534 \mathrm{~N}$ and $194 \mathrm{~N}$, respectively [12]. Hence, an increase of the MCL tension of more than $200 \mathrm{~N}$ after an osteotomy of $10^{\circ}$ is quite a substantial amount of force.

The MCL has been described as the primary static stabiliser against valgus rotation of the knee [7, 14]. In an OWO, a release of the superficial MCL is needed for exposure and, without a release, this results in a higher cartilage pressure in the medial compartment than in the lateral compartment $[1,8]$. However, a release of the superficial MCL results in a valgus instability $[11,13]$. There was also a significant increase in the valgus laxity. In the present study, a complete release of the superficial MCL was performed. Pape et al. [11] investigated the presence of valgus instability after partial versus complete release of the superficial MCL by measuring the medial joint opening on stress radiographs. They concluded that the anterior fibres of the superficial MCL play a crucial role in maintaining valgus stability. Therefore, the release of the superficial MCL for 
an OWO should be kept to a minimum to decrease the potential of late valgus instability.

The clinical consequence of valgus laxity after a release of the superficial MCL has been recently investigated in a study by Seo et al. [15]. They explored the changes in medial laxity of the knee joint after a complete release of the superficial MCL in patients who underwent an OWO, by measuring the medial joint space opening on radiographs before, during and after surgery. They found that a complete release of the superficial MCL during OWO increases the medial joint space opening. However, the medial joint space opening decreased to the level before the release of the superficial MCL after fixing with the TomoFix plate following the opening of the osteotomy site. No significant differences were found after 3-, 6- and 12-month follow-up. Gaasbeek et al. [4] investigated the valgus stability comparing an OWO with a closed-wedge osteotomy and found that the OWO group showed a mean postoperative decrease, and not an increase, of the mean MCL laxity of $4.5^{\circ}$ (SD 1.5) versus 5.3 (SD 1.2) in the closed-wedge osteotomy group $(p=0.04)$. However, they did not perform a release of the superficial MCL during the OWO; they shifted the superficial MCL and pes anserinus dorsally. The clinical results were equal between both groups after one-year follow-up [4]. After a follow-up of 7.8 years, there was no difference in survival rate between the OWO and closed-wedge osteotomy group [18].

A release of the superficial MCL has been shown to lead to valgus instability $[11,13]$. This is in line with the findings of the present study. However, there is a discrepancy between the biomechanical and clinical findings, as a postoperative valgus instability after an OWO and release of the superficial MCL is not a common complication [6]. There are several explanations for this discrepancy. Firstly, the muscular support of the dynamic stabilisers, such as the semimembranosus tendon and the medial head of the gastrocnemius, may partially compensate for the release of the superficial MCL [13]. Secondly, there could be a retensioning effect of the remaining fibres of the MCL, which could restore the valgus stability [10]. Thirdly, a tendonto-bone healing of the superficial MCL might occur during rehabilitation, thus preventing a (late) valgus instability [11].

There are several limitations to this study. Firstly, this was an experimental set-up; the measurements were not performed in vivo. Secondly, we performed our study without axial loading of the cadavers, which would have been more comparable with a clinical situation. Hence, the lateralisation of the axial force vector due to the osteotomy was not taken into account in this study. Nevertheless, even without axial loading, we found similar results to Agneskircher et al. [1], who performed a comparable study in cadaver knees, but with loading of the cadavers. Thirdly, we tested the valgus laxity only in extension. Although the MCL is the primary static stabiliser against valgus rotation of the knee, in extension the posterior medial capsule seems to be an important structure and in flexion it is the superficial MCL [3, 14]. Nevertheless, in extension, we found a significant increase in valgus laxity after release of the superficial MCL, so, in flexion, the valgus laxity is expected to be more pronounced. Fourthly, only the static stabilisers against valgus rotation were left intact, we resected all the dynamic stabilisers against valgus rotation, such as the semimembranosus tendon and the medial head of the gastrocnemius. As mentioned before, the dynamic stabilisers might partly compensate for the release of the superficial MCL. Ideally, this study would have been performed in a dynamic setting with all the stabilising structures intact. Fifthly, it is known that the tension in a ligament decreases over time with a constant strain $[5,16]$. The largest relaxation is within the first six to eight hours, after that, the effect is much smaller [5]. We only investigated the relaxation and the effect on the cartilage pressure in $5 \mathrm{~min}$, except for one knee, which we investigated for $24 \mathrm{~h}$. The pattern of relaxation in the present study was similar to that described in the literature $[5,16]$. It is expected that the relaxation of the MCL and the decrease in cartilage pressure would be higher after six to eight hours. Nevertheless, the cartilage pressure remained very high even after $24 \mathrm{~h}$. Finally, we resected the menisci to fit in the pressure-sensitive film in the medial and lateral tibiofemoral compartments. This might have influenced our results, possibly an overestimation of the (peak) CP and an underestimation of the CA. In 1986, Baratz et al. [2] had already studied the effects of meniscectomy on contact areas and the stresses in the knee joints of human cadavers using pressure-sensitive film. Loss of the medial meniscus led to a decrease in contact areas of approximately $75 \%$ and an increase in the peak contact pressures of approximately 235\%. Nevertheless, our results were comparable to Agneskircher et al. [1], who preserved the menisci.

The present study showed that a release of the superficial MCL is necessary for a successful OWO. Postoperatively, surgeons should consider the stability of the knee, as a release of the superficial MCL increased valgus laxity.

\section{Conclusion}

A release of the superficial MCL helps achieve the goal of reducing medial cartilage pressure in an OWO. A considerable relaxation of the MCL after an OWO occurred, which resulted in a decrease of the mean CP in the medial and lateral compartments of the knee over time. Cartilage pressure shifted from the medial to the lateral compartment only after a release of the superficial MCL. The release of 
the superficial MCL caused a significant increase in valgus laxity, which could influence stability after an OWO.

Author contributions NE participated in the study's design, carried out this study, and drafted the manuscript. GH performed the statistical analysis and drafted the manuscript. DJ participated in the study's design and helped in performing the investigations, AV carried out the study, NV participated in the study design, AK participated in the study design and helped in performing the investigations. All authors read and approved the final manuscript.

\section{Compliance with ethical standards}

Conflict of interest The authors declare that they have no conflict of interest.

Open Access This article is distributed under the terms of the Creative Commons Attribution 4.0 International License (http:// creativecommons.org/licenses/by/4.0/), which permits unrestricted use, distribution, and reproduction in any medium, provided you give appropriate credit to the original author(s) and the source, provide a link to the Creative Commons license, and indicate if changes were made.

\section{References}

1. Agneskirchner JD, Hurschler C, Wrann CD, Lobenhoffer $\mathrm{P}$ (2007) The effects of valgus medial opening wedge high tibial osteotomy on articular cartilage pressure of the knee: a biomechanical study. Knee Surg Sports Traumatol Arthrosc 23:852-861

2. Baratz ME, Fu FH, Mengato R (1986) Meniscal tears: the effect of meniscectomy and of repair on intraarticular contact areas and stress in the human knee. Am J Sports Med 14:270-274

3. Bruns J, Volkner M, Luessenhop S (1994) Pressure distribution in the knee joint. Influence of flexion with and without ligament dissection. Arch Orthop Trauma Surg 113:204-209

4. Gaasbeek RDA, Nicolaas L, Rijnberg WJ, van Loon CJM, van Kampen A (2010) Correction accuracy and collateral laxity in open versus closed wedge high tibial osteotomy. A one-year randomised controlled trial. Int Orthop 34:201-207

5. Kennedy JC, Hawkins RJ, Willis RB, Danylchuk KD (1976) Tension studies of human ligaments. J Bone Joint Surg 58-A:350-355

6. Lee DC, Byun SJ (2012) High tibial osteotomy. Knee Surg Relat Res 24(2):61-69

7. Liu F, Yue B, Gadikota HR, Kozanek M, Liu W, Gill TJ, Rubash HE, Li G (2010) Morphology of the medial collateral ligament of the knee. J Orthop Surg Res. 5:69

8. Lobenhoffer P, Agneskircher J, Zoch W (2004) Die öffnende valgisierende Osteotomie der proximalen Tibia mit Fixation durch einen medialen Plattenfixateur. Orthopäde 33:153-160
9. Mucha A, Dordevic M, Hirschmann A, Rasch H, Amsler F, Arnold MP, Hirschmann MT (2015) Effect of high tibial osteotomy on joint loading in symptomatic patients with varus aligned knees: a study using SPECT/CT. Knee Surg Sports Traumatol Arthrosc 23(8):2315-2323

10. Paley D, Bhatnagar J, Herzenberg JE, Bhave A (1994) New procedures for tightening knee collateral ligaments in conjunction with knee realignment osteotomy. Orthop Clin North Am 25(3):533-555

11. Pape D, Duchow J, Rupp S, Seil R, Kohn D (2006) Partial release of the superficial medial collateral ligament for openwedge high tibial osteotomy. A human cadaver study evaluating medial joint opening by stress radiography. Knee Surg Sports Traumatol Arthrosc 14:141-148

12. Robinson JR, Bull AMJ, Amis AA (2005) Structural properties of the medial collateral ligament complex of the human knee. J Biomechan 38:1067-1074

13. Robinson JR, Bull AMJ, Rhidian R de, Thomas W (2006) The role of the medial collateral ligament and posteromedial capsule in controlling knee laxity. Am J Sports Med 34(11):1815-1823

14. Robinson JR, Sanchez-Ballester J, Bull AMJ, Amis AA (2004) The posteromedial corner revisited: an anatomical description of the passive restraining structures of the medial aspect of the human knee. J Bone Joint Surg Br 86-B:674-681

15. Seo SS, Kim CW, Seo JH, Kim DH, Lee CR (2016) Does superficial medial collateral ligament release in open-wedge high tibial osteotomy for varus osteoarthritic knees increase valgus laxity? Am J Sports Med 44(4):908-915

16. Snijders CJ, Frankel V, Nordin M (2001) Biomechanica van het spierskeletstelsel: grondslagen en toepassingen. Springer Media B.V., Maarssen, pp 136-149

17. Suero EM, Sabbagh Y, Westphal R, Hawi N, Citak M, Wahl FM, Krettek C, Liodakis E (2015) Effect of medial opening wedge high tibial osteotomy on intraarticular knee and ankle contact pressure. J Orthop Res 33(4):598-604

18. van Egmond $\mathrm{N}$, van Grinsven $\mathrm{S}$, van Loon CJ, Gaasbeek RD, Kampen A (2016) Better clinical results after closed-compared to open-wedge high tibial osteotomy in patients with medial knee osteoarthritis. Knee Surg Sports Traumatol Arthrosc 24(1):34-41

19. Vrancken ACT, Eggermont F, van Tienen TG, Hannink G, Buma P, Janssen D, Verdonschot N (2016) Functional biomechanical performance of a novel anatomically shaped polycarbonate urethane total meniscus replacement. Knee Surg Sports Traumatol Arthrosc 24(5):1485-1494

20. Wilharm A, Hurschler C, Dermitas T, Bohnsack M (2013) Use of Tekscan K-scan sensors for retropatellar pressure measurement avoiding errors during implantation and the effects of shear forces on the measurement precision. Biomed Res Int. doi: $10.1155 / 2013 / 829171$ 